\title{
Activity and safety of temozolomide in advanced adrenocortical carcinoma patients
}

\author{
Deborah Cosentini 1,*, Giuseppe Badalamenti'2, \\ Andrea Spallanzani ${ }^{5}$, Paola Perotti ${ }^{3}$, Emanuela Musso ${ }^{2}$, Marta Laganà', Vittorio D Ferrari ${ }^{1}$, Gabriele Luppi ${ }^{5}$, \\ Alberto Dalla Volta', Lorena Incorvaia ${ }^{2}$, Sandra Sigala ${ }^{6}$, Antonio Russo ${ }^{2}$, Marco Volante ${ }^{4}$, Massimo Terzolo ${ }^{3}$ and \\ Alfredo Berruti ${ }^{1}$
}

${ }^{1}$ Medical Oncology Unit, Department of Medical and Surgical Specialties, Radiological Sciences, and Public Health, University of Brescia, ASST Spedali Civili, Brescia, Italy, ${ }^{2}$ Section of Medical Oncology, Department of Surgical, Oncological and Oral Sciences, University of Palermo, Palermo, Italy, ${ }^{3}$ Internal Medicine, Department of Clinical and Biological Sciences, San Luigi Hospital, University of Turin, Orbassano, Turin, Italy, ${ }^{4}$ Department of Oncology, San Luigi Hospital, University of Turin, Orbassano, Turin, Italy, ${ }^{5}$ Department of Medical Oncology, AOU di Modena, Modena, Italy, and ${ }^{6}$ Section of Pharmacology, Department of Molecular and Translational Medicine, University of Brescia, Brescia, Italy

Correspondence should be addressed to A Berruti

Email

alfredo.berruti@gmail.com

\begin{abstract}
Objective: Temozolomide has shown a significant anti-proliferative activity on adrenocortical cancer (ACC) cells in vitro. Design: On the basis of these results the drug was prescribed as second/third line in advanced metastatic ACC patients in four referral centers in Italy.

Methods: We retrospectively collected anagraphic, clinical and pathological data of patients with advanced ACC with disease progression to standard chemotherapy plus mitotane who were treated with temozolomide at the dose of $200 \mathrm{mg} / \mathrm{m}^{2} /$ die given for 5 consecutive days every 28 days. The primary endpoint was the disease control rate, defined as objective response or disease stabilization after 3 months. Secondary endpoints were overall survival (OS), progression-free survival (PFS) and drug safety.

Results: Twenty-eight patients have been included in the study. Ten patients (35.8\%, 95\% Cl: 17.8-53.8) obtained a disease control from temozolomide treatment. In particular, 1 patient had a complete response, 5 patients a partial response and 4 patients stable disease. Median PFS was 3.5 months and median OS was 7.2 months. Disease response was more frequently observed in patients with methylation of O6-methylguanine-DNA methyltransferase (MGMT) gene. Temozolomide therapy was well tolerated and most toxicities were limited to grade G1-2 according to WHO criteria.

Conclusion: Temozolomide was found active in the management of advanced ACC patients. The disease control rate obtained, however, was short-lived and the prognosis of treated patients was poor.
\end{abstract}

\section{Introduction}

Adrenocortical carcinoma (ACC) is a rare and aggressive tumor with an incidence of $0.7-2$ new cases per million populations per year (1). Surgery is the mainstay of therapy but a significant proportion of patients have non-resectable tumors at diagnosis; moreover, disease relapse occurs within 2 years in the majority of patients who undergo radical resection (1). This is the reason why adjuvant mitotane therapy is recommended by recent guidelines $(2,3)$, despite the low evidence of efficacy $(1$, $2,4,5,6)$.

The standard systemic treatment for advanced/ metastatic ACC patients, not eligible to surgery, is

Published by Bioscientifica Ltd. 
mitotane. This drug is administered either alone (1), or in combination with etoposide, doxorubicin and cisplatin (EDP-M regimen) (7). The efficacy of the EDP-M regimen, however, is limited as shown by the results of a randomized clinical trial reporting a disease response in about 25\% of treated patients with a median survival of 14 months (8). No effective therapies are available for patients with disease progression after EDP-M (9). The combination of gemcitabine and capecitabine, which is recommended by currently available guidelines $(2,3)$, is poorly efficacious $(10,11)$. Other treatment strategies, including modern molecular target therapies and immunotherapy (12, 13, 14), failed to demonstrate significant activity. New therapeutic options are therefore needed.

Temozolomide is an alkylating drug initially used in the treatment of brain tumors (15). The drug has been demonstrated to be efficacious also in the management of neuroendocrine tumors (16) and malignant pheochromocytoma/paraganglioma (17). Creemers and colleagues recently published a preclinical study exploring the activity of temozolomide in ACC cells in vitro. Their data showed that the drug has cytotoxic and cytostatic effects through a strong inhibition of cell growth, apoptosis and cell cycle arrest (18). Moreover, a weak association was found between the in vitro cytotoxicity of temozolomide and the epigenetic silencing of the MGMT (O6-methylguanine-DNA methyltransferase) DNA-repair (MGMT) gene, which is associated with temozolomide efficacy in patients with glioblastoma $(19,20,21)$ and advanced neuroendocrine tumors (16).

Given these results and the limited therapeutic strategies available in the management of advanced ACC, four Italian reference centers for this rare disease used temozolomide in ACC patients with disease progression to standard therapies. In this study, we did a retrospective assessment of temozolomide activity and toxicity in this peculiar clinical setting.

\section{Patients and methods}

\section{Study design and patients' characteristics}

This is a multicentric, retrospective study. Twenty-eight consecutive ACC patients treated with temozolomide from January 2016 to January 2018 at four Italian Institutions were included. Inclusion and exclusion criteria were considered also according to the summary of product characteristics of temozolomide. All patients met the following eligibility criteria: age >18 years; Eastern
Cooperative Oncology Group (ECOG) performance status 0-2; life expectancy of at least 3 months; pathological diagnosis of ACC; locally advanced or metastatic disease not suitable for surgery; at least one unidimensional (RECIST criteria) measurable lesion; adequate bone marrow reserve (neutrophils $\geq 1500 / \mathrm{mm}^{3}$ and platelets $\geq 100000 / \mathrm{mm}^{3}$, hemoglobin $\geq 9.0 \mathrm{~g} / \mathrm{dL}$ ); total bilirubin $\leq 1.5$ times the upper limit of normal; serum creatinine $\leq 1.5$ the upper limit of normal; effective contraception in premenopausal female and male patients. Exclusion criteria were history of prior malignancy, except for cured non-melanoma skin cancer, cured in situ cervical carcinoma, or other treated malignancies with no evidence of disease for at least 3 years; active clinically serious infections (greater than grade 2, Common Toxicity Criteria CTC V5.0); symptomatic metastatic brain or meningeal tumors; seizure disorder requiring medication (i.e. steroids or antiepileptics); decompensated heart failure (ejection fraction $\leq 45 \%$ ); myocardial infarction or revascularization procedure during the last 6 months; unstable angina pectoris; uncontrolled cardiac arrhythmia; hypertension not controlled by medications; pregnant or breast-feeding patients; prior treatment with temozolomide; other anticancer chemotherapy or immunotherapy during the study or within 4 weeks of study entry; radiotherapy during study or within 3 weeks of study start (palliative radiotherapy was allowed); major surgery within 4 weeks of study start; concomitant treatment with another investigational drug. Before starting therapy all patients were examined at the Medical Oncology of ASST-Spedali Civili in Brescia. After assessing eligibility for temozolomide therapy, the drug was prescribed and a written informed consent for offlabel temozolomide therapy was signed by each patient. Patients resident in other cities were referred to four referral oncology centers whose hospitals approved the off-label use of the drug. Temozolomide was distributed to the patients free of charge by the hospital pharmacy of all involved Institutions. The retrospective study was approved by the Ethical Review Board of ASST-Spedali Civili in Brescia (Protocol No. 3132).

Treatment consisted in temozolomide at the planned dose of $200 \mathrm{mg} / \mathrm{m}^{2} /$ die given for 5 consecutive days every 28 days. Maintenance of previous mitotane treatment was allowed but not mandatory and blood drug levels were monitored. Disease re-staging by CT scan and/or MRI was performed every three cycles. The following demographic, clinical and pathological data were collected: sex, age, medical history, physical examination, performance status, routine laboratory tests, endocrine work-up, chest 
and abdominal CT scan, other imaging data (i.e. brain $\mathrm{CT}$, MRI, bone scan) performed at baseline and during temozolomide treatment.

The study primary endpoint was the disease control rate, defined as the proportion of patients obtaining objective tumor response or stabilization at CT scan after 3 months of temozolomide therapy, using RECIST criteria, version 1.1. Secondary endpoints were the evaluation of progression-free survival (PFS), overall survival (OS) and treatment toxicity. PFS was defined as the time relapsing from the beginning of the treatment until disease progression or death whatever event occurred first. Nonprogressing patients still alive were censored at the last follow-up examination. Overall survival was defined as the time interval between the date of treatment start and the date of death from any cause or the last known alive date. The mENSAT classification and GRAS parameters (22) were used to assess prognosis. In particular, GRAS parameters were considered favorable if grading defined by Ki67 was $<20 \%$, primary R0 resection status attained, age $<50$ years and absence of symptoms at diagnosis; unfavorable in case of age $>50$ years, or presence of symptoms at diagnosis; pejorative in case of grading as defined by Ki67 $>20 \%$ and/or primary R1-2 resection status. The Common Toxicity Criteria CTC V5.0 were used to assess toxicity.

\section{Assessment of MGMT promoter methylation in tumor samples}

As ancillary study, we evaluated the predictive role of MGMT ( $\mathrm{O}^{6}$-methylguanine-DNA methyltransferase) promoter methylation in a patient subset included in this study for which tumor samples were available. MGMT promoter methylation status was performed by means of pyrosequencing technique. Ten methylated CpG sites were analyzed, located in the promoter region (NG_052673.1-chr10:131 265 507-131 265 556) of MGMT gene exon 1 and involved in the regulation of gene expression. Genomic DNA (gDNA) was extracted using FFPE tissues after manual microdissection, for neoplastic cell enrichment (at least 50\% of tumor cells), using the Maxwell ${ }^{\circledR}$ RSC instrument and tissue DNA Kit (Promega s.r.l). A total of $500 \mathrm{ng}$ of gDNA was modified by bisulfite conversion using a commercial available and certified CE-IVD kit (MGMT plus, Diatech Pharmacogenetics, Ancona, Italy) following manufacturer's instructions. Sequencing analysis was performed on PyroMark Q96MA apparatus (Biotage, Uppsala, Sweden) with PCR and sequencing primers supplied in the MGMT plus kit according to the manufacturer's instructions.

Data analysis was performed using the PyroMarkCpG software (Biotage), obtaining a mean percentage of the ten CpG methylated islands for each case. A cut-off of $5 \%$ (mean of the CpG islands) of methylation was used to define 'methylated' ( $>5 \%)$ and 'unmethylated' $(\leq 5 \%)$ samples. Methylated and unmethylated controls were properly used to take control of all workflow.

\section{Statistical analysis}

Descriptive statistics were used to analyze the patient clinical characteristics. Differences between categorical variables were assessed by a chi-square or the Fisher test when indicated. The PFS and OS curves were calculated with the Kaplan-Meier method and compared with the log-rank test. The primary end point of the study was to estimate the activity of the therapy in terms of proportion of patients attaining a clinical benefit. With 28 patients recruited, this study has a potency of $80 \%$ to refuse a clinical benefit rate of $15 \%$ (p0) and to assess the activity of the therapy as a disease control rate of $40 \%$; given an alpha error of 0.05. Statistical significance was set at $P<0.05$. SPSS $v 17.0$ software was used for the statistical analyses (SPSS Inc.).

\section{Results}

\section{Patients' characteristics}

The characteristics of the 28 enrolled patients are summarized in Table 1 . Median age at baseline was 54 years (range 31-72). Thirteen patients (46.4\%) had a hormone-secreting tumor at diagnosis and ten of them (35.7\%) had Cushing syndrome. Twenty-six patients $(92.8 \%)$ underwent primary surgery as the first treatment and 20 of them (71.4\%) obtained a complete resection (R0). Median disease-free survival of surgical treated patients was 19.9 months (range 5-49). Twelve patients (42.9\%) received postoperative adjuvant mitotane.

Cisplatin alone or the EDP combination regimen, both administered in association with mitotane, were the first-line cytotoxic therapies adopted after the diagnosis of metastatic disease. At baseline conditions, before starting temozolomide, the majority of patients (71.4\%) had a performance status $\leq 1$. According to mENSAT classification, 16 patients $(57.1 \%)$ had a stage IV-A disease, 9 (32.2\%) stage IV-B, 3 (10.7\%) stage IV-C. GRAS 
Table 1 Patients characteristics.

\begin{tabular}{|c|c|}
\hline Characteristics & Values \\
\hline Total number of patients & 28 \\
\hline Age, years & $54(31-72)$ \\
\hline \multicolumn{2}{|l|}{ Sex, $n(\%)$} \\
\hline Males & $17(60.7)$ \\
\hline Females & $11(39.3)$ \\
\hline Secreting at diagnosis, $n(\%)$ & $13(46.4)$ \\
\hline Cortisol excess & $10(35.7)$ \\
\hline Androgen excess & $3(10.7)$ \\
\hline Non secreting tumors & $15(53.6)$ \\
\hline \multicolumn{2}{|l|}{ Histological characteristics at diagnosis } \\
\hline Ki67, \% & $20(2-50)$ \\
\hline Ki67 <20\% & $15(53.6)$ \\
\hline $\mathrm{Ki} 67 \geq 20 \%$ & $13(46.4)$ \\
\hline Mitotic Rate (n/50HPF) & $10(2-66)$ \\
\hline Weiss Score & $6.5(3-9)$ \\
\hline \multicolumn{2}{|l|}{ GRAS*,$n(\%)$} \\
\hline Favorable & $6(21.4)$ \\
\hline Unfavorable & $9(32.2)$ \\
\hline Pejorative & $13(46.4)$ \\
\hline Primary surgery, $n(\%)$ & $26(92.8)$ \\
\hline RO & $20(71.4)$ \\
\hline Adjuvant mitotane, $n(\%)$ & $12(42.9)$ \\
\hline Disease free survival, months & $19.9(5-49)$ \\
\hline \multicolumn{2}{|l|}{ First-line metastatic treatment, $n(\%)$} \\
\hline EDP-M & $21(75)$ \\
\hline Cisplatin only & $7(25)$ \\
\hline \multicolumn{2}{|l|}{ Second-line metastatic treatment, $n(\%)$} \\
\hline Cisplatin only & $2(7.1)$ \\
\hline Gemcitabine plus capectitabine & $15(53.6)$ \\
\hline Mitotane in advanced setting, $n(\%)$ & $28(100)$ \\
\hline \multicolumn{2}{|l|}{ ECOG PS**,$n(\%)$} \\
\hline $0-1$ & $20(71.4)$ \\
\hline$>1$ & $8(28.6)$ \\
\hline \multicolumn{2}{|l|}{ mENSAT***,$n(\%)$} \\
\hline Stage IV-A & $16(57.1)$ \\
\hline Stage IV-B & $9(32.2)$ \\
\hline Stage IV-C & $3(10.7)$ \\
\hline
\end{tabular}

Data are expressed as median and range if not otherwise specified. *GRAS parameters are considered favorable if grading defined by Ki67 is $<20 \%$, primary R0 resection status performed, age $<50$ years and absence of symptoms at diagnosis. GRAS parameters are classified unfavorable in case of age $>50$ years or presence of symptoms at diagnosis. GRAS parameters are classified as pejorative in case of grading as defined by Ki67 $>20 \%$ and/or primary R1-2 resection status. **ECOG PS Performance Status sec ECOG assessed at the beginning of temozolomide therapy. ***mENSAT stage assessed at the beginning of temozolomide was defined as IV-A when tumor involves two organs, IV-B in case of three tumor organs, IV-C in case of $>3$ tumor organs.

parameters were favorable in $6(21.4 \%)$, unfavorable in 9 $(32.2 \%)$ and pejorative in $13(46.4 \%)$ patients.

\section{Treatment administered and activity}

Temozolomide was administered as second-line therapy in 11 patients (39.3\%), third-line therapy in 15 patients (53.6\%) and fourth-line approach in 2 (7.1\%). All patients continued mitotane therapy during temozolomide administration and the drug levels were within the therapeutic range in $10(35.7 \%)$ of them.

Median dose of temozolomide was $250 \mathrm{mg} / \mathrm{die}$ given for 5 consecutive days and median number of temozolomide cycles was 4 (range 2-16). One patient (3.6\%) obtained complete clinical response evaluated by RECIST 1.1 criteria, while partial response was observed in 5 patients (17.9\%), and 4 patients (14.3\%) obtained disease stabilization. However, 18 patients $(64.2 \%)$ had disease progression (Fig. 1). The overall response rate was $21.5 \%$ (95\% CI: 6.5-27.5) and a disease control was obtained in 10 patients (35.8\%) (95\% CI: 17.8-53.8) (Table 2).

At disease progression, further chemotherapy was administered in six patients; in particular, four patients received gemcitabine and capecitabine, whereas two patients received cisplatin as single agent.

\section{Patients' outcome}

Median PFS was 3.5 months (range: 1.2-24.2) (Fig. 2A) and OS was 7.5 months (range 2-38.8) (Fig. 2B). No significant difference in PFS was seen stratifying patients on the basis of whether they received temozolomide as second or further line of treatment (median PFS 3.9 months, range 1.4-24.2 vs 3.5 months, range 1.2-15.8, respectively,

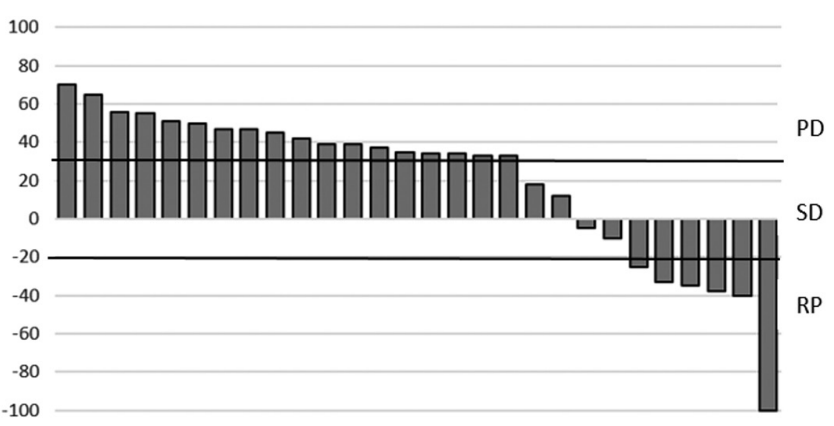

\section{Figure 1}

Waterfall plot of temozolomide responses. PD, progressive disease, defined by RECIST criteria as at least a $20 \%$ increase in the sum of the longest diameter (LD) of target lesions, taking as reference the smallest sum LD recorded since the treatment started or the appearance of one or more new lesions. SD, stable disease, defined by RECIST criteria as neither sufficient shrinkage to qualify for PR nor sufficient increase to qualify for PD, taking as reference the smallest sum LD since the treatment started. PR, partial response, defined by RECIST criteria as at least a 30\% decrease in the sum of the LD of target lesions, taking as reference the baseline sum LD. 
Table 2 Treatment response.

\begin{tabular}{lc}
\hline Complete response & $1(3.6 \%)$ \\
Partial response & $5(17.9 \%)$ \\
Stable disease & $4(14.3 \%)$ \\
Progressive disease & $18(64.2 \%)$ \\
Overall response rate & $6(21.5 \%, 95 \% \mathrm{Cl}: 6.5-27.5)$ \\
Clinical benefit & $10(35.8 \%, 95 \% \mathrm{Cl}: 17.8-53.8)$ \\
\hline
\end{tabular}

$P=0.62$ ). In addition, the attainment of a clinical benefit did not have any positive impact on OS: median 8.1 (range 3.6-38.8) in patients attaining a clinical benefit vs 7.1 months (range 2-33.3) in those who did not $(P=0.78)$.

Additional analyses were done to explore the potential prognostic significance of several patient and tumor characteristics. The patients with ECOG PS 0-1 had longer OS than those with PS 2 (median: 10.5 months, range: $2.6-38.8$ vs 3.2 months, range: $2.0-7.5$,
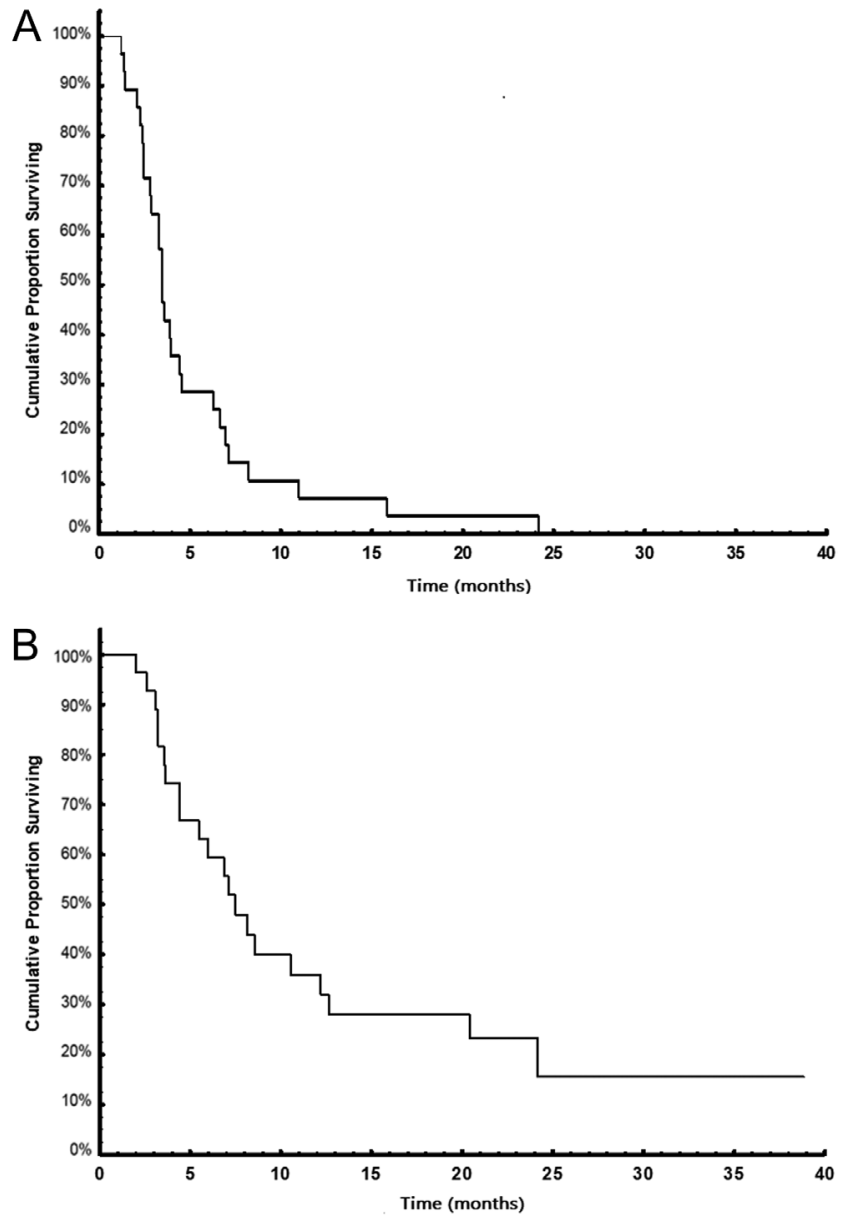

Figure 2

(A) Progression-free survival in patients treated with temozolomide. (B) Overall survival in patients treated with temozolomide. respectively, $P=0.001$ ) (Fig. 3A). Favorable GRAS score was associated with a longer OS (median: 12.2 months, range: 5.5-33.3) than unfavorable/pejorative GRAS (median: 6.9 months, range: 2.0-38.8), although the difference was not statistically significant $(P=0.17)$ (Fig. 3B). OS curves were similar in patients with unfavorable and pejorative GRAS score (data not shown). However, mENSAT failed to be associated with OS (mENSAT A: 7.5 months, range: 2.0-38.8, vs mENSAT B-C: 6.9 months, range: 2.6-33.3, $P=0.82)$; and the prognostic significance of mENSAT was not improved by the addition of GRAS (mENSAT A+GRAS A median OS: 12.2 months, range: 5.5-24.2, vs mENSAT + GRAS B-C: 7.5 months, range: 2.0-38.8,
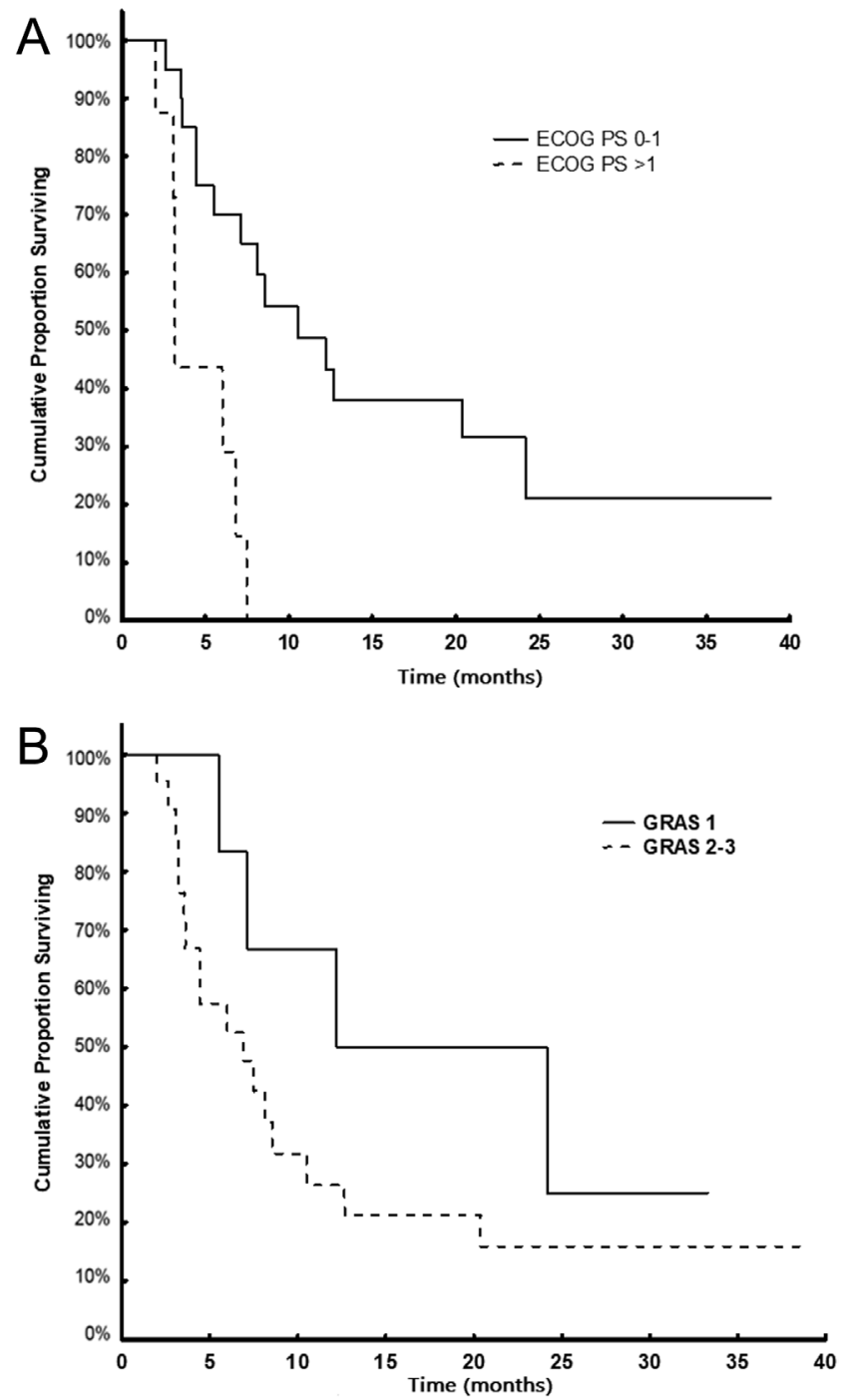

Figure 3

(A) Overall survival on the basis of ECOG performance status $(P 0.001)$. (B) Overall survival on the basis of GRAS parameters: favorable vs unfavorable or pejorative ( $P$ 0.17). 
$P=0.56$ ) (data not shown). Finally, stratifying patients according to mitotane plasma concentrations, PFS and OS were 4.4 months (range: $2.9-24.2$ ) and 12.7 months (range: 4.4-33.3), respectively in patients in which blood mitotane was within the therapeutic range (14-20 $\mu \mathrm{g} / \mathrm{L}$ ), while they were 3.3 months (range: 2.4-15.8) and 6.9 months (range: 2.4-38.8) respectively in those in which the drug was below $14 \mu \mathrm{g} / \mathrm{L}(P=0.36$ for PFS, $P=0.37$ for OS) (Fig. 4).

\section{Treatment toxicity}

Patients were evaluated after each cycle with both clinical examination and blood chemistry (complete blood count, liver and renal function). The observed temozolomide toxicities are summarized in Table 3. As expected, nausea and vomiting were the most frequent side effects, occurring in 35.8 and $25 \%$ of patients, respectively. These symptoms were classified as grade 3 in $14.3 \%$ and in $10.7 \%$ of patients, respectively. Liver toxicity was observed in $25 \%$ of patients, being grade 3 in $7.2 \%$ of cases. Only 1 patient (3.6\%) developed grade 3 neutropenia. The other observed toxicities were renal impairment and thrombocytopenia, involving 25\% and $21.4 \%$ of patients, respectively. A 1-week delay in the start of a new cycle was needed in 12 patients due to toxicity, whereas no dose reduction was prescribed.

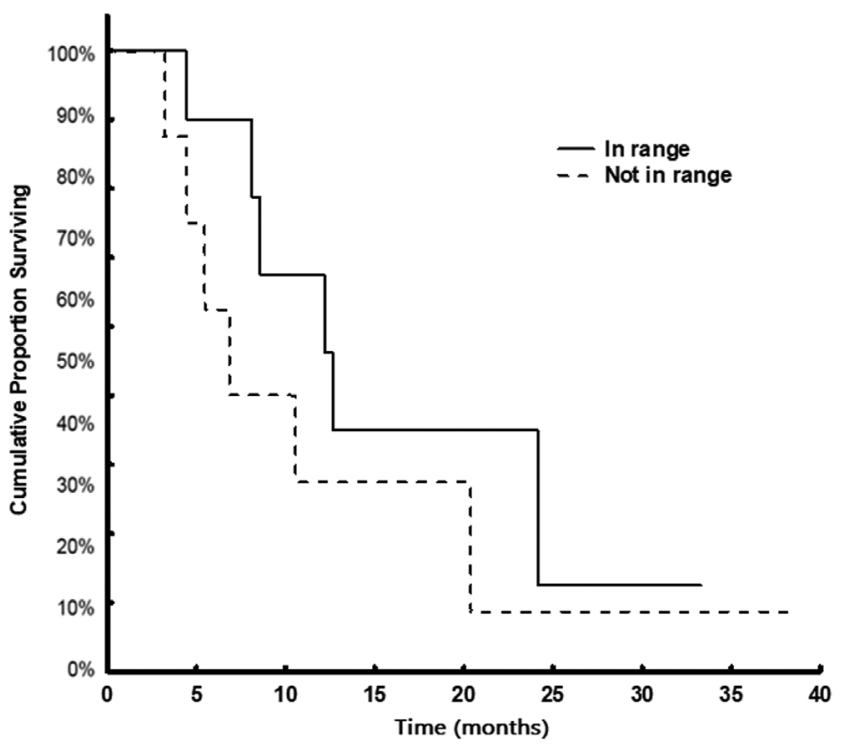

\section{Figure 4}

Overall survival on the basis of circulating mitotane levels (P 0.37).

\section{MGMT status and relevant correlation with drug activity}

MGMT status was evaluated in 15 patients, eight of them showing MGMT promoter methylation. In the methylated MGMT group, 4 patients out of $8(50.0 \%)$ obtained a disease response, in particular, 1 had a complete response and 3 had a partial response. Conversely, in the nonmethylated MGMT group, only 1 patient out of 7 (14.3\%) had a partial response, whereas 1 patient (14.3\%) had a stable disease and the others had progressive disease (Table 4). No differences in PFS or OS were observed comparing patients with (median PFS: 4.4 months, range: 2.1-24.2; median OS: 6.9 months, range: 3.1-12.7, respectively) or without (median PFS: 3.9 months, range: 2.1-15.8; median OS: not reached, range: 3.2-38.8) MGMT methylation ( $P=0.55, P=0.08$ respectively) (data not shown).

\section{Discussion}

Alkylating agents have shown some activity in ACC. Our group observed a clinical benefit of the administration of oral cyclophosphamide on a metronomic schedule in two heavily pre-treated patients (23). Moreover, streptozotocyn (Sz) demonstrated a response rate of 35\% in 40 advanced ACC assessed retrospectively in Sweden (24). On the basis of these results, Sz was considered as the best treatment to be tested against EDP in the FIRM-ACT trial (8). Since this combination appeared inferior to EDP, $\mathrm{Sz}$ is not generally used as first-line approach. However, it is still recommended as a possible second-line therapy by currently available guidelines $(2,3)$.

Due to the demonstrated activity of alkylating agents in the management of ACC and the preclinical findings showing that temozolomide exerted a potent antitumor effect on ACC cells in vitro (18), four Italian reference centers obtained the authorization for the offlabel administration of the drug at dose of $200 \mathrm{mg} / \mathrm{m}^{2} /$ die in ACC patients with disease progression after firstline chemotherapy with cisplatin-containing regimens plus mitotane. In this retrospective evaluation of these patients, temozolomide was as a whole well tolerated and toxicities were manageable. In particular, toxicities were less frequent than previously seen in other tumors, except for thrombocytopenia which was found in a higher number of patients (21.4 vs $13.7 \%)(16,25)$.

Tumor responses according to RECIST criteria were observed in $20 \%$ of patients, whereas about $35 \%$ had a 
Table 3 Temozolomide toxicity.

\begin{tabular}{|c|c|c|c|c|}
\hline & \multicolumn{4}{|c|}{ Toxicity, $\boldsymbol{n}=\mathbf{2 8}(100 \%)$} \\
\hline & All grades & G1 & $\mathrm{G} 2$ & G3 \\
\hline Nausea & $10(35.8 \%)$ & $4(14.3 \%)$ & $2(7.2 \%)$ & $4(14.3 \%)$ \\
\hline Vomiting & $7(25 \%)$ & $1(3.6 \%)$ & $3(10.7 \%)$ & $3(10.7 \%)$ \\
\hline Liver toxicity & $7(25 \%)$ & $4(14.3 \%)$ & $1(3.6 \%)$ & $2(7.2 \%)$ \\
\hline Renal toxicity & $7(25 \%)$ & $4(14.3 \%)$ & $3(10.7 \%)$ & $0(0 \%)$ \\
\hline Thrombocytopenia & $6(21.4 \%)$ & $4(14.3 \%)$ & $2(7.2 \%)$ & $0(0 \%)$ \\
\hline Neutropenia & $5(17.9 \%)$ & $2(7.1 \%)$ & $2(7.2 \%)$ & $1(3.6 \%)$ \\
\hline
\end{tabular}

disease control. Although comparisons between different trials have to be considered with caution, these results seem to be better in terms of response rate than those obtained by the association gemcitabine plus capecitabine (about 4\%) while similar data were seen in terms of disease control rate $(10,11)$. Unfortunately, disease response and stabilization to temozolomide were shortlived and did not influence patients survival, which was only of 7 months on average. This finding is in contrast to what it is commonly observed in chemotherapy trials, in which disease response usually discriminates two patient populations with different prognosis (26).

In these pre-treated ACC patients, poor performance status was the strongest negative prognostic factor, whereas mENSAT stage failed to correlate with PFS and OS. Favorable GRAS score was associated with better outcome in terms of PFS and OS without attaining the statistical significance than either unfavorable and pejorative scores. The low number of patients enrolled conferred to our series a low potency to test these prognostic factors.

It was found in other malignancies that the therapeutic benefit of temozolomide depends on its ability to alkylate/ methylate DNA. This methylation damages the DNA, thus triggering cell death by apoptosis. Primary resistance to temozolomide in glioblastoma and in neuroendocrine tumors is often directly related to high MGMT expression $(16,19)$. So MGMT inactivation by methylation is a marker of temozolomide tumor cell sensitivity. In our series, we were able to assess MGMT expression in 15 cases. Despite the low numbers, the objective response rate was of $50 \%$ in methylated ACC vs $14 \%$ in non-methylated ACC, and this finding is consistent with the potential role of MGMT inactivation in favoring temozolomide cytotoxicity in ACC patients.

This study provides for the first time data on activity of temozolomide in ACC patients, the retrospective design and the small number of patients included are the main limitations.

In conclusion, temozolomide, administered as second-line approach in ACC, induced a significant tumor shrinkage in about one out of five patients. Despite this non-negligible activity, the drug failed to demonstrate to be efficacious, since the responses observed were shortlived and did not influence patient survival. On the basis of these data, we believe that temozolomide should not be recommended as second-line therapy in unselected ACC

Table 4 MGMT methylation assessment.
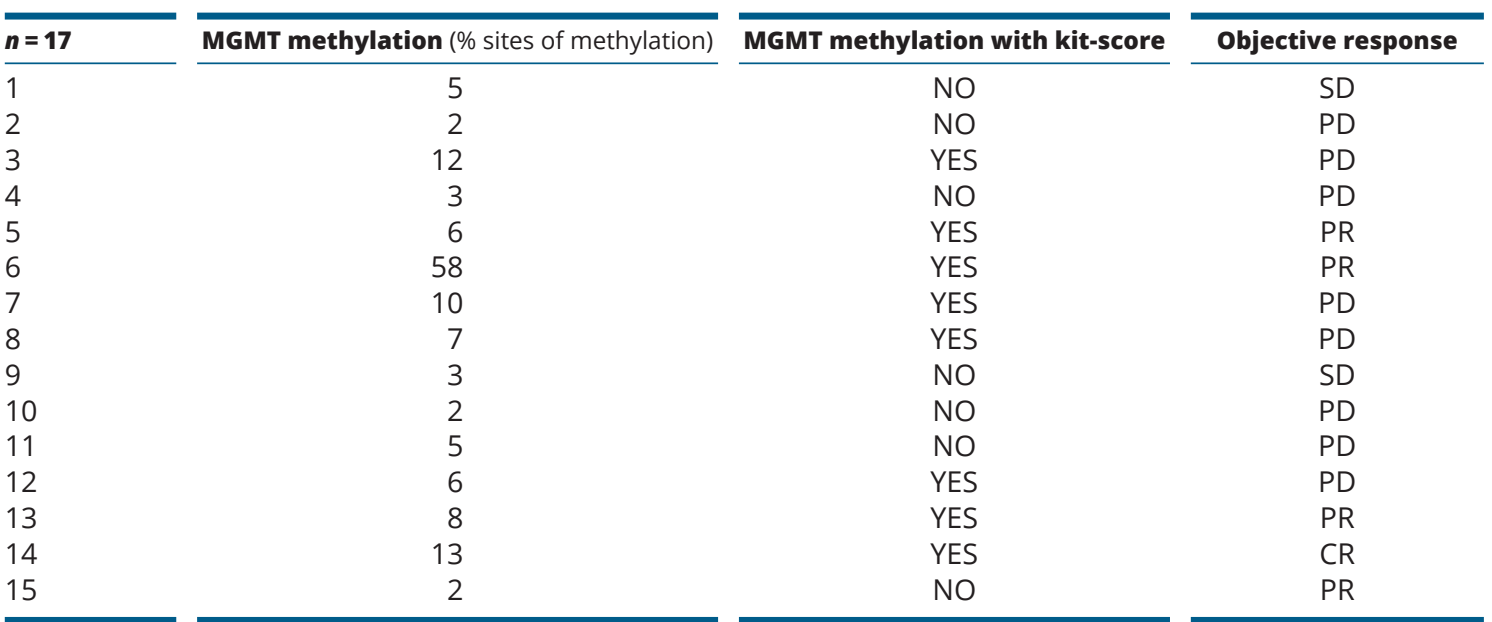

\begin{tabular}{rrrr}
\hline \multicolumn{1}{c}{ OS } & & \multicolumn{1}{c}{ PFS } \\
\cline { 1 - 1 } 38.8 & & 15.8 \\
33.3 & & 7.0 \\
12.7 & & 6.6 \\
3.2 & & 2.5 \\
3.1 & & 2.1 \\
8.6 & & 4.5 \\
12.2 & & 2.9 \\
6.0 & & 7.1 \\
8.1 & & 3.5 \\
20.4 & & 3.9 \\
20.5 & & 3.9 \\
6.9 & & 2.8 \\
4.4 & & 4.4 \\
24.2 & & 24.2 \\
5.1 & & 2.1 \\
\hline
\end{tabular}

$\mathrm{CR}$, complete response; PD, progressive disease; PR, partial response; SD, stable disease; UND, undetermined, objective response. 
patients. However, the drug could be a potential option in patients with good PS bearing MGMT methylated ACC, and this may represent a first step toward a personalized medicine approach in advanced ACC.

\section{Declaration of interest}

The authors declare that there is no conflict of interest that could be perceived as prejudicing the impartiality of this study.

\section{Funding}

This this study was supported in part by FIRM onlus, Cremona, Italy.

\section{Acknowledgements}

This research was presented in part as a poster at the ENS@T Meeting, 2018.

\section{References}

1 Terzolo M, Daffara F, Ardito A, Zaggia B, Basile V, Ferrari L \& Berruti A. Management of adrenal cancer: a 2013 update. Journal of Endocrinological Investigation 201437 207-217. (https://doi. org/10.1007/s40618-013-0049-2)

2 Fassnacht M, Dekkers OM, Else T, Baudin E, Berruti A, de Krijger RR, Haak HR, Mihai R, Assie G \& Terzolo M. European Society of Endocrinology Clinical Practice Guidelines on the management of adrenocortical carcinoma in adults, in collaboration with the European Network for the Study of Adrenal Tumors. European Journal of Endocrinology 2018179 G1-G46. (https://doi.org/10.1530/EJE-180608)

3 Berruti A, Baudin E, Gelderblom H, Haak HR, Porpiglia F, Fassnacht M, Pentheroudakis G \& ESMO Guidelines Working Group. Adrenal cancer: ESMO Clinical Practice Guidelines for diagnosis, treatment and follow-up. Annals of Oncology 201223 (Supplement 7) vii131-vii138. (https://doi.org/10.1093/annonc/mds231)

4 Berruti A, Grisanti S, Pulzer A, Claps M, Daffara F, Loli P, Mannelli M, Boscaro M, Arvat E, Tiberio G et al. Long-term outcomes of adjuvant mitotane therapy in patients with radically resected adrenocortical carcinoma. Journal of Clinical Endocrinology and Metabolism 2017102 1358-1365. (https://doi.org/10.1210/jc.2016-2894)

5 Terzolo M, Baudin AE, Ardito A, Kroiss M, Leboulleux S, Daffara F, Perotti P, Feelders RA, deVries JH, Zaggia B et al. Mitotane levels predict the outcome of patients with adrenocortical carcinoma treated adjuvantly following radical resection. European Journal of Endocrinology 2013169 263-270. (https://doi.org/10.1530/EJE-130242)

6 Berruti A, Fassnacht M, Haak H, Else T, Baudin E, Sperone P, Kroiss M, Kerkhofs T, Williams AR, Ardito A et al. Prognostic role of overt hypercortisolism in completely operated patients with adrenocortical cancer. European Urology 201465 832-838. (https://doi.org/10.1016/j. eururo.2013.11.006)

7 Berruti A, Terzolo M, Sperone P, Pia A, Della Casa S, Gross DJ, Carnaghi C, Casali P, Porpiglia F, Mantero F et al. Etoposide, doxorubicin and cisplatin plus mitotane in the treatment of advanced adrenocortical carcinoma: a large prospective phase II trial. Endocrine-Related Cancer 200512 657-666. (https://doi.org/10.1677/ erc.1.01025)

8 Fassnacht M, Terzolo M, Allolio B, Baudin E, Haak H, Berruti A, Welin S, Schade-Brittinger C, Lacroix A, Jarzab B et al. Combination chemotherapy in advanced adrenocortical carcinoma. New England
Journal of Medicine 2012366 2189-2197. (https://doi.org/10.1056/ NEJMoa1200966)

9 Megerle F, Kroiss M, Hahner S \& Fassnacht M. Advanced adrenocortical carcinoma - what to do when first-line therapy fails? Experimental and Clinical Endocrinology and Diabetes 2019127 109-116. (https://doi.org/10.1055/a-0715-1946)

10 Sperone P, Ferrero A, Daffara F, Priola A, Zaggia B, Volante M, Santini D, Vincenzi B, Badalamenti G, Intrivici C et al. Gemcitabine plus metronomic 5-fluorouracil or capecitabine as a second-/ third-line chemotherapy in advanced adrenocortical carcinoma: a multicenter phase II study. Endocrine-Related Cancer 201017 445-453. (https://doi.org/10.1677/ERC-09-0281)

11 Henning JEK, Deutschbein T, Altieri B, Steinhauer S, Kircher S, Sbiera S, Wild V, Schlötelburg W, Kroiss M, Perotti P et al. Gemcitabine-based chemotherapy in adrenocortical carcinoma: a multicenter study of efficacy and predictive factors. Journal of Clinical Endocrinology and Metabolism 2017102 4323-4332. (https://doi. org/10.1210/jc.2017-01624)

12 Konda B \& Kirschner LS. Novel targeted therapies in adrenocortical carcinoma. Current Opinion in Endocrinology, Diabetes, and Obesity 201623 233-241. (https://doi.org/10.1097/MED.0000000000000247)

13 Le Tourneau C, Zarwan C, Hoimes C, Wong DJ, Bauer S, Wermke M, Grote HJ, von Heydebreck A, Chin K \& Gulley J. Avelumab in patients with metastatic adrenocortical carcinoma (mACC): results from the JAVELIN solid tumor trial. Annals of Oncology 201728 (Supplement 5) mdx371.067. (https://doi.org/10.1186/s40425-0180424-9)

14 Cosentini D, Grisanti S, Dalla Volta A, Laganà M, Fiorentini C, Perotti P, Sigala S \& Berruti A. Immunotherapy failure in adrenocortical cancer: where next? Endocrine Connections 20187 E5-E8. (https://doi.org/10.1530/EC-18-0398)

15 Ma W, Li N, An Y, Zhou C, Bo C \& Zhang G. Effects of temozolomide and radiotherapy on brain metastatic tumor: a systematic review and meta-analysis. World Neurosurgery 201692 197-205. (https://doi. org/10.1016/j.wneu.2016.04.011)

16 Koumarianou A, Kaltsas G, Kulke MH, Oberg K, Strosberg JR, Spada F, Galdy S, Barberis M, Fumagalli C, Berruti A et al. Temozolomide in advanced neuroendocrine neoplasms: pharmacological and clinical aspects. Neuroendocrinology 2015101 274-288. (https://doi. org/10.1159/000430816)

17 Hadoux J, Favier J, Scoazec JY, Leboulleux S, Al Ghuzlan A, Caramella C, Déandreis D, Borget I, Loriot C, Chougnet C et al. SDHB mutations are associated with response to temozolomide in patients with metastatic pheochromocytoma or paraganglioma. International Journal of Cancer 2014135 2711-2720. (https://doi.org/10.1002/ ijc.28913)

18 Creemers SG, van Koetsveld PM, van den Dungen ES, Korpershoek E, van Kemenade FJ, Franssen GJ, de Herder WW, Feelders RA \& Hofland LJ. Inhibition of human adrenocortical cancer cell growth by temozolomide in vitro and the role of the MGMT gene. Journal of Clinical Endocrinology and Metabolism 2016101 4574-4584. Erratum in: Journal of Clinical Endocrinology and Metabolism 2018103 3115-3118. (https://doi.org/10.1210/jc.2016-2768)

19 Esteller M, Garcia-Foncillas J, Andion E, Goodman SN, Hidalgo OF, Vanaclocha V, Baylin SB \& Herman JG. Inactivation of the DNArepair gene MGMT and the clinical response of gliomas to alkylating agents. New England Journal of Medicine 2000343 1350-1354. (https://doi.org/10.1056/NEJM200011093431901) Erratum in: New England Journal of Medicine 20003431740.

20 Hegi ME, Diserens AC, Godard S, Dietrich PY, Regli L, Ostermann S, Otten P, Van Melle G, de Tribolet N \& Stupp R. Clinical trial substantiates the predictive value of O-6-methylguanine-DNA methyltransferase promoter methylation in glioblastoma patients treated with temozolomide. Clinical Cancer Research 2004101871 1874. (https://doi.org/10.1158/1078-0432.CCR-03-0384) 
21 Hegi ME, Diserens AC, Gorlia T, Hamou MF, de Tribolet N, Weller M, Kros JM, Hainfellner JA, Mason W, Mariani L et al. MGMT gene silencing and benefit from temozolomide in glioblastoma. New England Journal of Medicine 2005352 997-1003. (https://doi. org/10.1056/NEJMoa043331)

22 Baudin E \& Endocrine Tumor Board of Gustave Roussy. Adrenocortical carcinoma. Endocrinology and Metabolism Clinics of North America 2015 44 411-434. (https://doi.org/10.1016/j.ecl.2015.03.001) Review Erratum in: Endocrinology and Metabolism Clinics of North America 201544 xix

23 Ferrero A, Sperone P, Ardito A, Rossi G, Del Buono S, Priola AM, Bracarda S, Taberna E, Terzolo M \& Berruti A. Metronomic chemotherapy may be active in heavily pre-treated patients with metastatic adreno-cortical carcinoma. Journal of Endocrinological Investigation 201336 148-152. (https://doi.org/10.3275/8334)
24 Khan TS, Imam H, Juhlin C, Skogseid B, Gröndal S, Tibblin S, Wilander E, Oberg K \& Eriksson B. Streptozocin and o,p'DDD in the treatment of adrenocortical cancer patients: long-term survival in its adjuvant use. Annals of Oncology 200011 1281-1287. (https://doi. org/10.1023/a:1008377915129)

25 Bae SH, Park MJ, Lee MM, Kim TM, Lee SH, Cho SY, Kim YH, Kim YJ, Park CK \& Kim CY. Toxicity profile of temozolomide in the treatment of 300 malignant glioma patients in Korea. Journal of Korean Medical Science 201429 980-984. (https://doi.org/10.3346/ jkms.2014.29.7.980)

26 Anderson JR, Cain KC \& Gelber RD. Analysis of survival by tumor response and other comparisons of time-to-event by outcome variables. Journal of Clinical Oncology 200826 3913-3915. (https:// doi.org/10.1200/JCO.2008.16.1000)

Received 23 July 2019

Revised version received 18 October 2019

Accepted 22 October 2019 